\title{
Salusins protect myocardium against ischemic injury by alleviating endoplasmic reticulum stress
}

\author{
WANG JianFei ${ }^{2,4 \dagger}$, WANG Yin ${ }^{3 \dagger}$, SHAN ShiFu ${ }^{2}$, HU TianTian ${ }^{2}$, CHEN HuYan ${ }^{2}$, \\ TIAN Jing ${ }^{1}$, REN AnJing ${ }^{1}$, ZHOU Xu $^{2}$, YUAN WenJun ${ }^{1,2 *} \&$ LIN Li $^{1 *}$ \\ ${ }^{1}$ Department of Physiology and Key Laboratory of Molecular Neurobiology of Ministry of Education, Second Military \\ Medical University, Shanghai 200433, China; \\ ${ }^{2}$ Department of Physiology and Neurobiology, Basic Medical College, Ningxia Medical University, Yinchuan 750004, China; \\ ${ }^{3}$ Department of Ultrasound, Changhai Hospital, Shanghai 200433, China; \\ ${ }^{4}$ Department of Gynecology and Obstetrics, Shanghai Punan Hospital, Shanghai 200125, China
}

Received October 27, 2011; accepted February 1, 2012

\begin{abstract}
Salusins are regulatory peptides that affect cardiovascular function. We previously reported that salusin- $\alpha$ and $-\beta$ protected cultured cardiomyocytes from serum deprivation-induced cell death through upregulating glucose-regulated protein 78 (GRP78), an endoplasmic reticulum (ER) resident protein whose overexpression acts as a marker and suppressor of ER stress. The present study examined whether salusin- $\alpha$ and $-\beta$ inhibit ER stress in ischemic myocardium. In a rat model of myocardial infarction created by ligating the left anterior descending coronary artery (LAD), salusin- $\alpha$ or $-\beta$ was intravenously injected at 5 or $15 \mathrm{nmol} \mathrm{kg}{ }^{-1} 15 \mathrm{~min}$ prior to $2 \mathrm{~h}$ of LAD occlusion. The high dose of salusin- $\alpha$ and $-\beta$ significantly improved heart function and hemodynamics in LAD-occluded rats, but had no effects in sham-operated rats. The arrhythmias caused by LAD occlusion were markedly attenuated by salusin- $\alpha$ and $-\beta$. The apoptotic rate in ischemic myocardium was reduced from $31.5 \% \pm 3.7 \%$ to $19.8 \% \pm 2.2 \%$ and $12.3 \% \pm 2.2 \%$, and the infarct size was reduced from $53.4 \% \pm 4.0 \%$ of the risk area to $26.5 \% \pm 9.7 \%$ and $23.7 \% \pm 8.9 \%$ by $15 \mathrm{nmol} \mathrm{kg}^{-1}$ salusin- $\alpha$ and $-\beta$, respectively. Furthermore, salusin- $\alpha$ and $-\beta$ prevented the activation of GRP78 and ER stress-specific apoptotic effectors caspase-12 and CHOP (C/EBP homologous protein), and attenuated the reduction of an ER stress-associated antiapoptotic protein Bcl-2 in ischemic cardiac tissue. The salusins also inhibited the ER stress induced by tunicamycin in cultured rat H9c2 cardiomyocytes. These results indicate that salusins protect myocardium against ischemic injury by inhibiting ER stress and ER stress-associated apoptosis.
\end{abstract}

salusin, myocardial ischemia, endoplasmic reticulum stress, rats

Citation: Wang J F, Wang Y, Shan S F, et al. Salusins protect myocardium against ischemic injury by alleviating endoplasmic reticulum stress. Sci China Life Sci, 2012, 55: 358-366, doi: 10.1007/s11427-012-4311-1

Salusin- $\alpha$ and $-\beta$ are endogenous bioactive peptides processed from preprosalusin, an alternatively spliced product of the torsion dystonia-related gene (TOR2A) [1]. Immunoreactive salusin- $\alpha$ and $-\beta$ are detected in a variety of human and murine tissues, serum, and urine [1-5]. A few studies have shown that salusins regulate cardiovascular function $[1,6,7]$. Intravenous administration of salusin- $\alpha$ and $-\beta$ to

$\dagger$ Contributed equally to this work

*Corresponding author (email: lilincn@hotmail.com; yuanwj@hotmail.com)

(C) The Author(s) 2012. This article is published with open access at Springerlink.com rats causes profound hypotension and bradycardia via negative cardiac inotropic and chronotropic actions [1,6]. Treatment of cultured neonatal rat cardiac myocytes with salusin- $\alpha$ and $-\beta$ promotes cell growth [7].

We previously reported that salusin- $\beta$ inhibited L-type calcium currents in adult rat cardiac myocytes [8], which may partially explain its negative inotropic effect $[1,6]$. Additionally, the inhibition of calcium influx by salusin- $\beta$ may potentially prevent intracellular calcium overload and con- 
sequent tissue injury caused by myocardial ischemia, a condition in which calcium overload is believed to be a major cause of cardiac dysfunction [9]. In another study in cultured neonatal rat cardiac myocytes [10], we found that salusin- $\alpha$ and $-\beta$ reduced cell death resulting from serum withdrawal by upregulating the expression of glucoseregulated protein 78 (GRP78), a chaperone protein that facilitates protein folding in the endoplasmic reticulum (ER). The induction of GRP78 is a specific marker and an endogenous survival response to ER stress, a condition of disrupted ER homeostasis due to the accumulation of unfolded and misfolded proteins in the ER lumen [11]. Previous studies have demonstrated the involvement of ER stress in myocardial ischemia [12-14]. Our previous observation of GRP78 induction by salusin- $\alpha$ and $-\beta$ in cardiac myocytes [10] raised the possibility that salusin- $\alpha$ and $-\beta$ may limit the damage of ER stress to myocytes. The present study tested the hypothesis that salusin- $\alpha$ and $-\beta$ protect against myocardial ischemia by relieving ER stress.

\section{Material and methods}

\subsection{Rat model of acute myocardial ischemia}

The model of acute myocardial ischemia in rats was prepared as described previously [15]. Briefly, male Sprague-Dawley rats (260-330 g, purchased from SIPPR-BK Laboratory Animal Co. Ltd., Shanghai, China) were anesthetized with sodium pentobarbitone $\left(60 \mathrm{mg} \mathrm{kg}^{-1}\right.$, intraperitoneally) and performed with tracheotomy. The femoral vein was cannulated for the administration of drugs and the femoral artery was cannulated to measure the arterial blood pressure (BP). Mean arterial pressure (MAP) and heart rate (HR) were calculated from the BP trace and a standard limb lead I ECG, respectively. A catheter was introduced into the left ventricle via the right carotid artery to obtain the left ventricular pressure (LVP) signals. LV systolic pressure (LVSP) and $\pm \mathrm{d} P / \mathrm{d} t_{\max }$ were calculated from the continuously recorded LVP signals. A left thoracotomy was performed and artificial respiration was immediately initiated with room air using a stroke volume of $1.5-2 \mathrm{~mL} / 100 \mathrm{~g}$ and a rate of 60 strikes min $^{-1}$ to maintain the arterial $\mathrm{PCO}_{2}, \mathrm{PO}_{2}$ and $\mathrm{pH}$ within the normal range [16]. After the heart was exposed, a 6/0 braided silk suture was placed around the left anterior descending coronary artery (LAD), approximately $3 \mathrm{~mm}$ away from the starting point of the LAD. The rats were allowed to stabilize for $15 \mathrm{~min}$, then salusin or vehicle was intravenously injected and LAD was occluded $15 \mathrm{~min}$ later. Myocardial ischemia was confirmed by the immediate ST-segment depression of the ECG, a decrease in blood pressure and visual cyanosis of the topical heart.

All animal procedures were approved by the Second Military Medical University, China and were in accordance with the Guide for Care and Use of Laboratory Animals published by the US NIH (publication No. 96-01).

\subsection{Intravenous administration of salusins}

The lyophilized powder of salusin- $\alpha$ or $-\beta$ (Phoenix Pharmaceuticals, Burlingame, CA, USA) was freshly dissolved in $100 \mu \mathrm{L}$ normal saline and intravenously injected at 5 or $15 \mathrm{nmol} \mathrm{kg}{ }^{-1} 15 \mathrm{~min}$ prior to the LAD occlusion. For control rats, the same volume of normal saline $(100 \mu \mathrm{L})$ was injected.

\subsection{Analyses of ischemic arrhythmias}

All the ventricular arrhythmias during $2 \mathrm{~h}$ occlusion of the LAD in rats were analyzed according to the guidelines of the Lambeth Conventions [17] and scored (arrhythmia score, AS) with Johnston's criterion [18]. Briefly, the arrhythmias were identified as a single ventricular ectopic beat (VEB), salvo (couplet or triplet VEBs), ventricular tachycardia (VT, a run of four or more consecutive VEBs) and ventricular fibrillation (VF). Each type of arrhythmia was quantified by counts and/or duration. The total VEBs were calculated as the sum of the individual VEBs in the rats that survived the 2-h ischemia. Rats that did die of irreversible VF were excluded from the arrhythmia analysis but included in the mortality calculation.

\subsection{Apoptosis analysis}

After $2 \mathrm{~h}$ of LAD occlusion, the heart was removed, fixed in formalin and cryosectioned with the thickness of each section set at $10 \mu \mathrm{m}$. To determine apoptosis, heart cryosections were stained with the terminal deoxynucleotidyltransferase-mediated dUTP nick-end labeling (TUNEL) reagents (C1086, Beyotime, Jiangsu, China), counterstained with Hoechst 33258 and analyzed under fluorescence microscopy.

\subsection{Infarct size measurement}

The infarct size following $2 \mathrm{~h}$ myocardial infarction was measured using the method of triphenyl-tetrazolium chloride (TTC) staining, as described previously [19]. First, 1\% Evan's blue dye was infused into the heart through the apex to mark the risk zone as unstained (not blue) tissue. Second, the hearts were quickly frozen and transversely cut into 2-mm thick slices. Third, the slices were incubated in $1 \%$ TTC (Sigma-Aldrich Corp., St. Louis, MO, USA) at $37^{\circ} \mathrm{C}$ for $15 \mathrm{~min}$ and fixed in $4 \%$ paraformaldehyde to enhance the contrast of the Evan's blue and TTC staining. The variable tissue stained red with TTC, while the dead tissue (infarct tissue) did not stain. Finally, the volumes of the infarct area and the risk area in each of the sections were calculated by ImageJ, a public domain image analysis program developed by the National Institutes of Health, USA. The infarct size was calculated as a percentage of the infarct area (white area) versus the risk area (non-blue area). 


\subsection{ER stress model in cultured rat H9c2 cardiomyo- cytes}

The H9c2 rat ventricular cell line was purchased from the Cell Bank of the Chinese Academy of Sciences. Cells were seeded at a concentration of $2 \times 10^{4}$ cells $\mathrm{cm}^{-2}$ and cultured with Dulbecco's Modified Eagle Medium (DMEM, Sigma) containing 10\% (v/v) fetal bovine serum (FBS, HyClone Laboratories, Logan, UT, USA) in a humidified atmosphere of $95 \%$ air $/ 5 \% \mathrm{CO}_{2}$ at $37^{\circ} \mathrm{C}$. When the cells reached $80 \%$ confluence, they were serum deprived for $12 \mathrm{~h}$ for synchronization, incubated with salusin- $\alpha$ or $-\beta$ for 30 min and then exposed to an ER stress inducer tunicamycin $\left(10 \mu \mathrm{g} \mathrm{mL}^{-1}\right)$ for $12 \mathrm{~h}$.

\subsection{Determination of the expression of ER stress-asso- ciated proteins}

The expression levels of ER stress markers GRP78, caspase-12 and CHOP (C/EBP homologous protein), and an ER stress-associated antiapoptotic protein Bcl-2 were determined by Western blot analysis. Briefly, heart tissue was homogenized and cultured cardiomyocytes were lysed in RIPA buffer (Beyotime), sonicated on ice and with the protein concentration determined using a bicinchoninic acid kit (Beyotime). Twenty micrograms of total proteins were electrophoresed on an SDS-PAGE gel, transferred onto a nitrocellulose membrane and blocked with 5\% non-fat dried milk. The membrane was then probed with a primary antibody followed by the peroxidase-conjugated secondary antibody and the signal was visualized using chemiluminescence reagents (Pierce, Rockford, IL, USA) and scanned with a Gene Gnome Syngene Bio Imaging system. The primary antibodies against GRP78, Bcl-2 and GAPDH were purchased from Santa Cruz Biotechnology (Santa Cruz, CA, USA), and the primary antibodies against caspase- 12 and CHOP were purchased from Cell Signaling Technology, Inc. (Danvers, MA, USA). The amount of proteins was quantified by densitometry and normalized to GAPDH, an internal standard.

\subsection{Statistical analyses}

The data of heart function and hemodynamic measurements, including MAP, HR, LVSP and $\pm \mathrm{d} P / \mathrm{d} t_{\max }$, were expressed as means $\pm \mathrm{SD}$. The differences between the control and salusin groups were analyzed with the repeated-measure ANOVA followed by the Fisher's least significant difference (LSD) test, and the differences between the pre- and post-occlusion time points within the same group were analyzed with the one-way ANOVA followed by the Dunnet test.

The incidences of single VEB, salvo, VT, reversible VF and mortality were expressed as percentages and compared between groups by Fisher's exact test. Arrhythmia counts and durations of VT/VF were expressed as the median (first quartile, third quartile) and compared between groups by the Mann-Whitney nonparametric test.

The data of apoptosis, infarct size and protein expression levels were expressed as means $\pm \mathrm{SD}$, and analyzed with the one-way ANOVA followed by the LSD test to determine the differences between groups. Two-tailed $P<0.05$ was considered statistically significant.

\section{Results}

\subsection{Improvement of heart function and hemodynamics by salusins in LAD-occluded rats}

The heart function and hemodynamic parameters including MAP, HR, LVSP, $+\mathrm{d} P / \mathrm{d} t_{\max }$ and $-\mathrm{d} P / \mathrm{d} t_{\max }$ were continuously recorded before and after LAD occlusion (Figure 1). The values of these parameters were comparable between all the groups before LAD occlusion. However, significant reductions of MAP, LVSP and $\pm \mathrm{d} P / \mathrm{d} t_{\max }$ were observed immediately after LAD occlusion in all the groups. These parameters increased to some extent after $5 \mathrm{~min}$, but failed to return to the level prior to occlusion during the observation period of $2 \mathrm{~h}$. No significant changes were observed for HR after LAD occlusion (data not shown).

In the LAD-occluded rats, the treatment with a high dose $(15 \mathrm{nmol} \mathrm{kg}-1)$ of salusin- $\alpha$ or $-\beta$ significantly improved MAP, LVSP and $\pm \mathrm{d} P / \mathrm{d} t_{\text {max }}\left(P<0.05\right.$ for MAP and $\pm \mathrm{d} P / \mathrm{d} t_{\max }$, $P<0.01$ for LVSP), but the low dose $\left(5 \mathrm{nmol} \mathrm{kg}^{-1}\right)$ of salusin- $\alpha$ or $-\beta$ did not affect any of the parameters (Figure 1). In addition, neither dose of salusins affected the heart function and hemodynamics in sham-operated rats (data not shown).

\subsection{Salusins reduced ischemic arrhythmias in LAD- occluded rats}

Before LAD occlusion, no arrhythmia occurred in any of the rats throughout the surgery and injection of salusins. After LAD occlusion, ischemic arrhythmias occurred in each of the rats, and most occurred in the first $30 \mathrm{~min}$. The representative episodes of ischemic arrhythmias are shown in Figure 2 and all the arrhythmias during $2 \mathrm{~h}$ occlusion of LAD are summarized in Table 1. A mortality of $28.6 \%$ resulting from irreversible VF was observed in LAD-occluded rats injected with normal saline. By contrast, no death occurred in rats injected with salusin- $\alpha$ or $-\beta$. LAD occlusion induced single VEBs, salvos and VT in all of the rats, whereas the high dose $\left(15 \mathrm{nmol} \mathrm{kg}{ }^{-1}\right)$ of salusin- $\beta$ significantly reduced the incidences of VT. Additionally, salusin- $\beta$ totally abolished the occurrence of VF. The arrhythmia counts, especially those of VT, and the overall arrhythmia score were dose-dependently reduced following treatment 

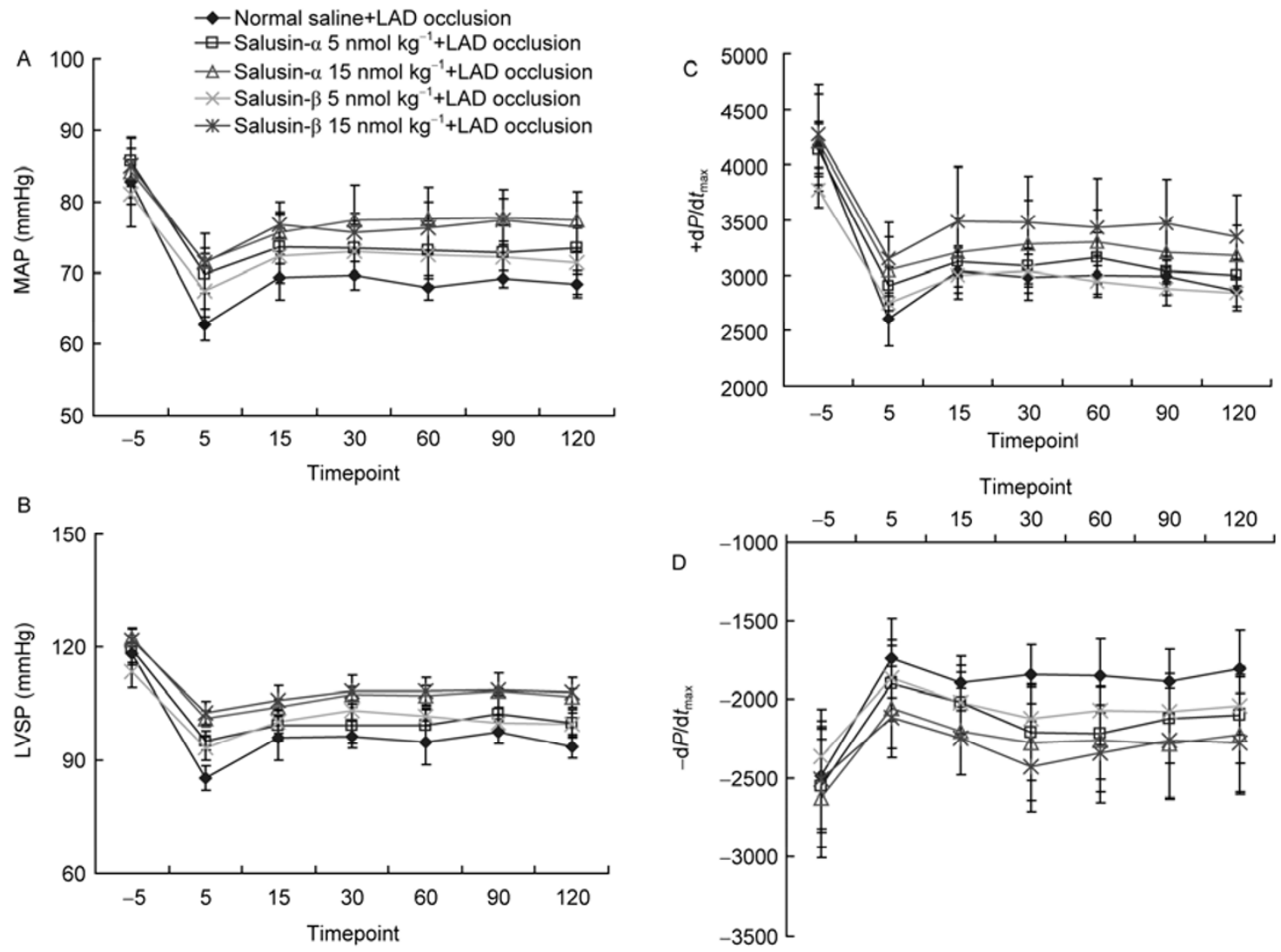

Figure 1 The changes of mean arterial pressure (MAP), left ventricular systolic pressure (LVSP), $+\mathrm{d} P / \mathrm{d} t_{\max }$ and $-\mathrm{d} P / \mathrm{d} t_{\max }$ after the occlusion of the LAD in rats. LAD occlusion was set as the zero time-point. "-5 min" indicates pre-occlusion and the positive numbers indicate post-occlusion. The time-point of "5 min" shows the maximum changes, which occurred within $5 \mathrm{~min}$ after LAD occlusion. Values are means $\pm \mathrm{SD}$ ( $n=7-8$ for each group). The statistical analysis of the repeated-measure ANOVA showed that salusin- $\alpha$ or $-\beta$ at dosages of $15 \mathrm{nmol} \mathrm{kg}{ }^{-1}$ significantly improved the MAP, LVSP and $\pm \mathrm{d} P / \mathrm{d} t_{\max }$ after LAD occlusion.

Table 1 Salusins reduced ischemic arrhythmias that occurred during $2 \mathrm{~h}$ occlusion of LAD in rats ${ }^{\mathrm{a})}$

\begin{tabular}{|c|c|c|c|c|c|c|c|c|c|c|c|c|}
\hline \multirow[b]{2}{*}{ Treatment } & \multicolumn{4}{|c|}{ Incidence $(\%)$} & \multirow[b]{2}{*}{$\begin{array}{l}\text { Mortality } \\
(\%)\end{array}$} & \multirow[b]{2}{*}{$\begin{array}{l}\text { Arrhythmia } \\
\text { score (AS) }\end{array}$} & \multicolumn{4}{|c|}{ Arrhythmia counts } & \multicolumn{2}{|c|}{ Duration (s) } \\
\hline & $\begin{array}{l}\text { Single } \\
\text { VEBs }\end{array}$ & Salvos & VT & Rev. VF & & & $\begin{array}{l}\text { Single } \\
\text { VEBs }\end{array}$ & Salvos & VT & Total VEBs & VT & Rev. VF \\
\hline $\begin{array}{l}\text { normal saline+LAD oc- } \\
\text { clusion }(n=14)\end{array}$ & 100 & 100 & 100 & 57.1 & 28.6 & $5.14 \pm 0.29$ & $\begin{array}{c}134 \\
(120,233)\end{array}$ & $24(11,39)$ & $\begin{array}{c}1165 \\
(669,1751)\end{array}$ & $\begin{array}{c}1323 \\
(976,1994)\end{array}$ & $\begin{array}{c}118 \\
(66,201)\end{array}$ & $\begin{array}{c}33 \\
(8,50)\end{array}$ \\
\hline $\begin{array}{c}\text { Salusin- } \alpha 5 \mathrm{nmol} \mathrm{kg}^{-1} \\
+\mathrm{LAD} \text { occlusion } \\
(n=8)\end{array}$ & 100 & 100 & 100 & 25 & 0 & $3.75 \pm 0.31^{* *}$ & $\begin{array}{c}165 \\
(126,351)\end{array}$ & $61(24,79)^{*}$ & $\begin{array}{c}420 \\
(236,1061)^{*}\end{array}$ & $\begin{array}{c}643 \\
(435,1601)\end{array}$ & $\begin{array}{c}40 \\
(21,111)^{*}\end{array}$ & $\begin{array}{c}0 \\
(0,8)^{* *}\end{array}$ \\
\hline $\begin{array}{c}\text { Salusin- } \alpha 15 \mathrm{nmol} \mathrm{kg}^{-1} \\
+ \text { LAD occlusion } \\
(n=8)\end{array}$ & 100 & 100 & 100 & $12.5^{*}$ & 0 & $3.25 \pm 0.31^{* *}$ & $\begin{array}{c}110 \\
(83,167)\end{array}$ & $23(14,56)$ & $\begin{array}{c}145 \\
(75,613)^{* *}\end{array}$ & $\begin{array}{c}344 \\
(261,918)^{* *}\end{array}$ & $\begin{array}{c}14 \\
(7,57)^{* *}\end{array}$ & $\begin{array}{c}0 \\
(0,0)^{* *}\end{array}$ \\
\hline $\begin{array}{c}\text { Salusin- } \beta 5 \mathrm{nmol} \mathrm{kg}{ }^{-1} \\
+ \text { LAD occlusion } \\
(n=8)\end{array}$ & 100 & 100 & 100 & $0^{*}$ & 0 & $2.63 \pm 0.18^{* *}$ & $\begin{array}{c}144 \\
(123,161)\end{array}$ & $10(4,25)$ & $\begin{array}{c}254 \\
(55,407)^{* *}\end{array}$ & $\begin{array}{c}426 \\
(168,593)^{* *}\end{array}$ & $\begin{array}{c}25 \\
(5,40)^{* *}\end{array}$ & $(0,0)^{* *}$ \\
\hline $\begin{array}{l}\text { Salusin- } \beta 15 \text { nmol kg-1 } \\
+ \text { LAD occlusion }(n=8)\end{array}$ & 100 & 100 & $62.5^{*}$ & $0^{*}$ & 0 & $1.75 \pm 0.16^{* *}$ & $\begin{array}{c}99 \\
(59,138)\end{array}$ & $6(3,12)^{*}$ & $\begin{array}{c}30 \\
(0,157)^{* * *}\end{array}$ & $\begin{array}{c}147 \\
(110,331)^{* * *}\end{array}$ & $\begin{array}{c}3 \\
(0,17)^{\text {*** }}\end{array}$ & $\begin{array}{c}0 \\
(0,0)^{* *}\end{array}$ \\
\hline
\end{tabular}

a) Abbreviations: VEBs, ventricular ectopic beats; VT, ventricular tachycardia; VF, ventricular fibrillation; Rev. VF, reversible VF. $*, P<0.05$; $* *$, $P<0.01$ vs. normal saline+LAD occlusion.

with salusin- $\alpha$ or $-\beta$. Compared to salusin- $\alpha$, salusin- $\beta$ showed more potent effects in reducing VT, and consequently reduced the total arrhythmia counts and arrhythmia score to a larger extent.

\subsection{Salusins inhibited apoptosis in the ischemic myo- cardium of LAD-occluded rats}

Significant apoptosis $(31.5 \% \pm 3.7 \%)$ was detected by the 

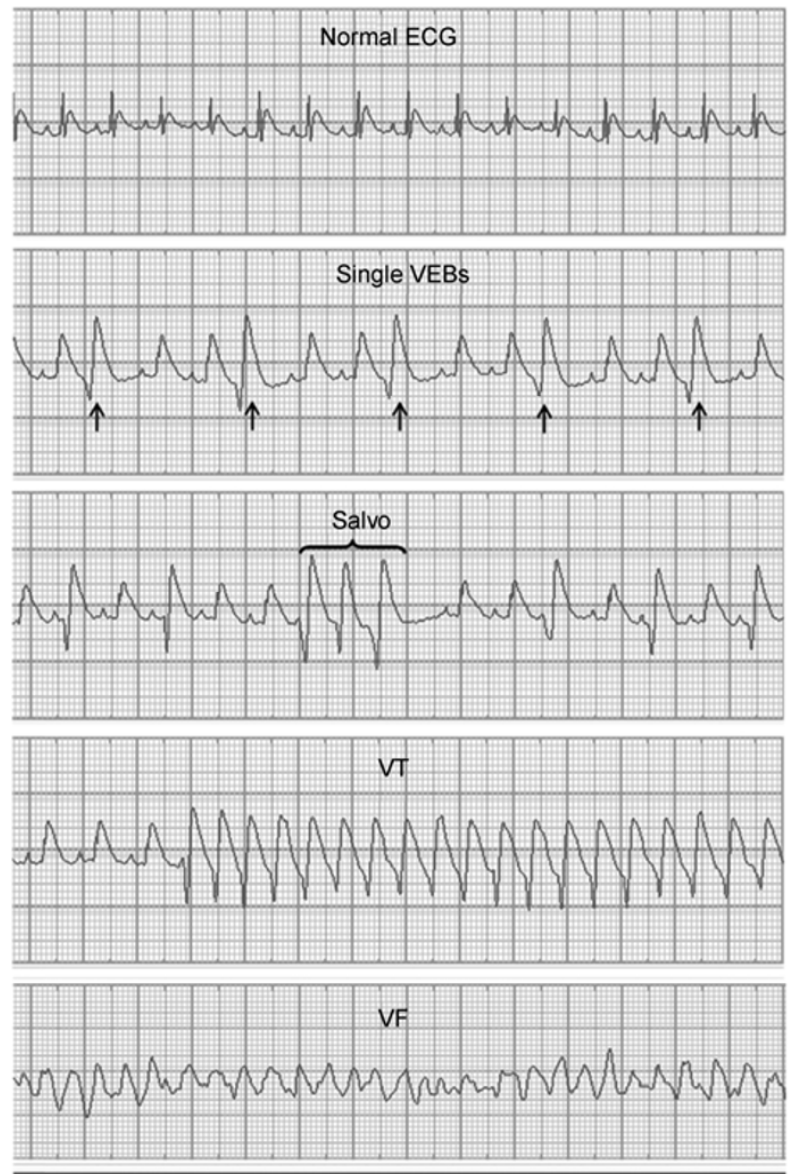

Figure 2 Representative recordings of normal ECG in rats before LAD occlusion and ischemic arrhythmias during LAD occlusion, including single ventricular ectopic beats (VEBs, indicated by arrows), salvo (couplet or triplet VEBs), ventricular tachycardia (VT, a run of four or more consecutive VEBs) and ventricular fibrillation (VF).

TUNEL assay in heart cryosections after $2 \mathrm{~h}$ of LAD occlusion. Salusin- $\alpha$ and $-\beta\left(15 \mathrm{nmol} \mathrm{kg}{ }^{-1}\right)$ reduced the apoptotic rate to $19.8 \% \pm 2.2 \%$ and $12.3 \% \pm 2.2 \%$, respectively (both $P<0.01)$, indicating that the apoptosis caused by myocardial ischemia was significantly inhibited by salusins (Figure 3).

\subsection{Salusins reduced infarct size in LAD-occluded rats}

The infarct size was measured in hearts after $2 \mathrm{~h}$ of LAD occlusion. As shown in Figure 4, both salusin- $\alpha$ and $-\beta$ decreased the infarct size. At the high dose of $15 \mathrm{nmol} \mathrm{kg}^{-1}$, salusin- $\alpha$ and $-\beta$ markedly decreased the infarct size from $53.4 \% \pm 4.0 \%$ of the risk area in the control rats to $26.5 \% \pm 9.7 \%$ and $23.7 \% \pm 8.9 \%$, respectively (both $P<0.01$ ).

\subsection{Salusins attenuated ER stress in the ischemic myo- cardium of LAD-occluded rats}

In the ischemic myocardium after $2 \mathrm{~h}$ of LAD occlusion, the protein levels of GRP78 (a marker of ER stress), the cleaved form of active caspase-12 (an ER stress-specific
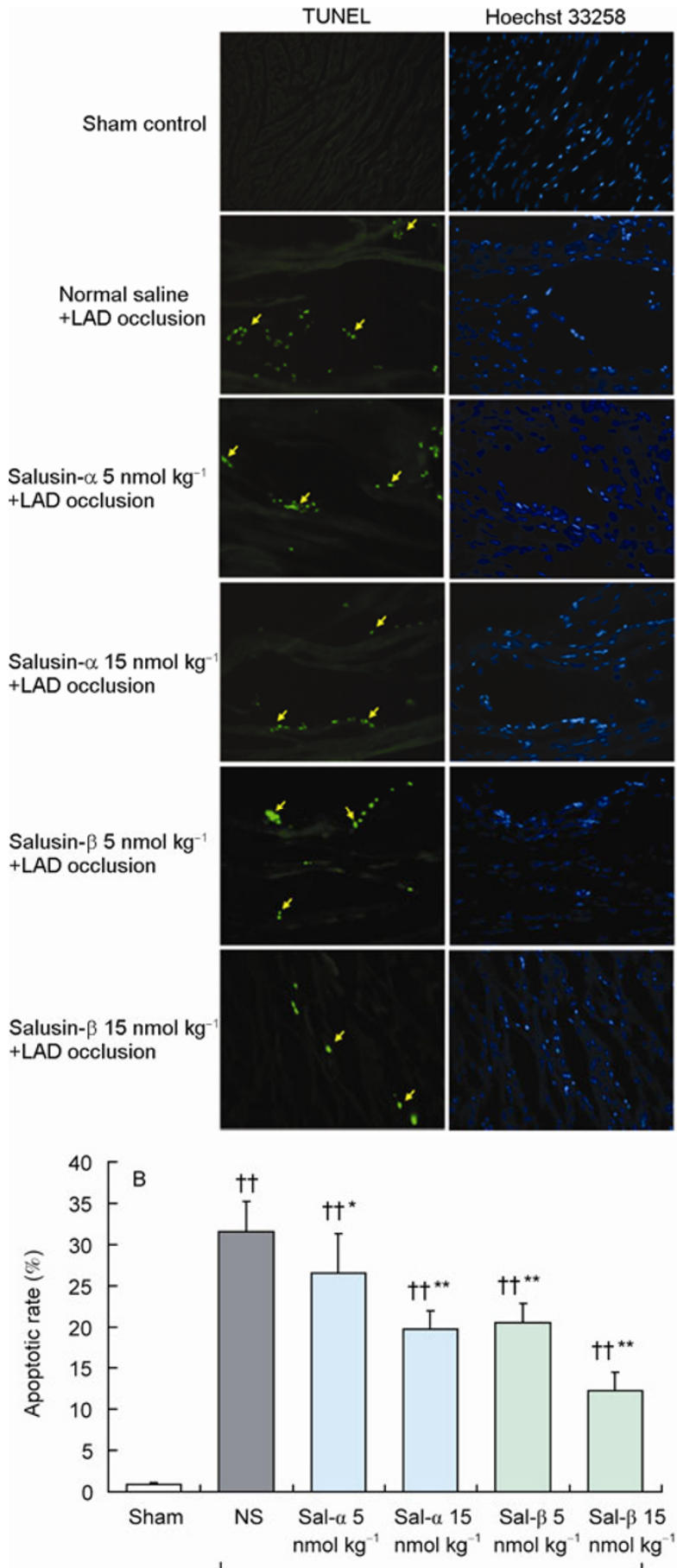

+ LAD occlusion

Figure 3 Salusins reduced apoptosis, as detected by a TUNEL assay, in heart cryosections after $2 \mathrm{~h}$ of LAD occlusion. A, Representative images of TUNEL staining and Hoechst 33258 counterstaining (magnification 400x). Arrows indicate typical apoptotic myocytes. B, Quantitative analysis of apoptotic cells. Values are means $\pm \mathrm{SD}$ ( $n=4$ for each group). $\dagger, P<0.05$; $\dagger \dagger$, $P<0.01$ vs. sham; *, $P<0.05$; **, $P<0.01$ vs. normal saline (NS)+LAD occlusion.

caspase) and CHOP (ER stress-specific transcription factor) markedly increased by $2.8,3.9$ and 7.1 fold, respectively. In contrast, the expression level of Bcl-2, an antiapoptotic 


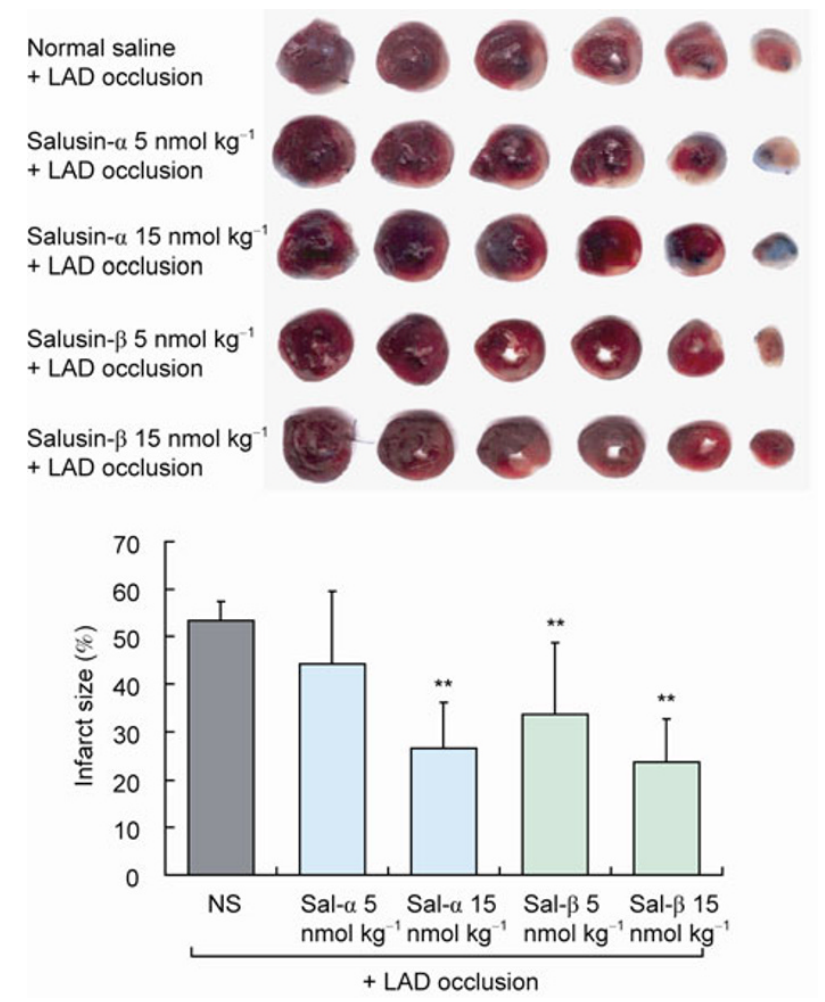

Figure 4 Salusins reduced infarct size in rats subjected to $2 \mathrm{~h}$ of LAD occlusion. A, Representative images of serial heart sections stained with Evan's blue and TTC. B, Quantitative analysis of infarct size, expressed as a percentage volume of the infarct area versus the risk area. Values are means \pm SD ( $n=5-9$ for each group). *, $P<0.05$; **, $P<0.01$ vs. normal saline (NS)+LAD occlusion.

protein associated with ER stress, was significantly reduced to $34.8 \% \pm 5.9 \%$ of the control level. The treatment of either salusin- $\alpha$ or $-\beta$ at a dose of $15 \mathrm{nmol} \mathrm{kg} \mathrm{kg}^{-1}$ exclusively downregulated the protein levels of GRP78, active caspase-12 and CHOP, and significantly upregulated the expression of $\mathrm{Bcl}-2$, suggesting the attenuation of ER stress and ER stress-associated apoptosis (Figure 5).

\subsection{Salusins attenuated ER stress caused by tunicamy- cin in cultured cardiomyocytes}

In cultured rat $\mathrm{H} 9 \mathrm{c} 2$ cardiomyocytes, the ER stress inducer tunicamycin $\left(10 \mu \mathrm{g} \mathrm{mL} \mathrm{m}^{-1}\right)$ significantly induced ER stress. The protein levels of GRP78 and the cleaved form of active caspase- 12 were observed to significant increase by 3.1 and 15.5 fold, respectively. The treatment of salusin- $\alpha$ or $-\beta$ significantly downregulated both proteins, suggesting the direct inhibitory effects of salusins on ER stress (Figure 6).

\section{Discussion}

The endoplasmic reticulum (ER) is a centrally located intracellular organelle performing critical functions in protein synthesis and folding, lipid and sterol synthesis, and calcium homeostasis. Perturbation of any of the major functions of the ER results in ER stress. Such stress can be induced by various pathophysiological stimuli such as hypoxia, glucose deprivation and calcium depletion from the lumen [11]. ER stress is characterized by the accumulation of unfolded and misfolded proteins in the ER lumen that triggers the unfolded protein response (UPR), a highly conserved signaling system [11]. The UPR attempts to relieve ER stress by suppressing protein synthesis, upregulating protein-folding chaperones such as GRP78, and degrading misfolded proteins. If unsuccessful, the UPR can trigger apoptosis in stressed cells [20,21]. Previous studies have shown the involvement of ER stress in myocardial ischemia [12-14]. The present study first showed that salusin- $\alpha$ and $-\beta$ attenuated ER stress in the ischemic cardiac tissue of coronary-ligated rats, accompanied by reduced apoptosis, arrhythmias and infarct size, and improved heart function and hemodynamics. These observations indicate that salusin- $\alpha$ and $-\beta$ protect ischemic heart through relieving ER stress. We also showed that salusin- $\alpha$ and $-\beta$, at the same dose that reduced myocyte death in our previous observation [10], prevented the ER stress induced by tunicamycin, suggesting the direct inhibition of ER stress by salusins.

GRP78 is a well-characterized indicator of UPR activation, as well as a critical modulator of ER stress. It senses misfolded protein accumulation and dissociates from ER transmembrane proteins IRE1 (inositol-requiring kinase), PERK (dsRNA-activated protein kinase-like ER kinase) and ATF6 (activating transcription factor), which initiate the major signaling pathways of UPR [22]. The ATF6 pathway induces the expression of more than 30 different genes including GRP78, which allow cells to cope with ER stress [22]. When ER stress is prolonged or excessive, the UPR switches from a cytoprotective to an apoptotic response involving activation of specific proteins such as caspase 12 and CHOP. Caspase-12 is a murine protein associated with the cytoplasmic face of the ER membrane. It is activated only by ER stress-initiated apoptotic pathways [20]. CHOP is a proapoptotic transcription factor that is expressed at very low levels under physiological conditions but strongly induced during severe ER stress. CHOP promotes apoptosis in several ways, including transcriptionally downregulating the antiapoptotic protein Bcl-2, upregulating DR5, a member of the death receptor family, depleting cellular glutathione and increasing reactive oxygen species (ROS) production [20].

In accordance with previous studies [13,14], the present study observed that GRP78 expression was upregulated in the ischemic area of the left ventricle, concomitant with activation of ER stress-specific proapoptotic molecules caspase-12 and CHOP, and reduced expression of the antiapoptotic protein Bcl-2 (Figure 3), indicating the induction of ER stress and ER stress-specific apoptosis by myocardial ischemia. 

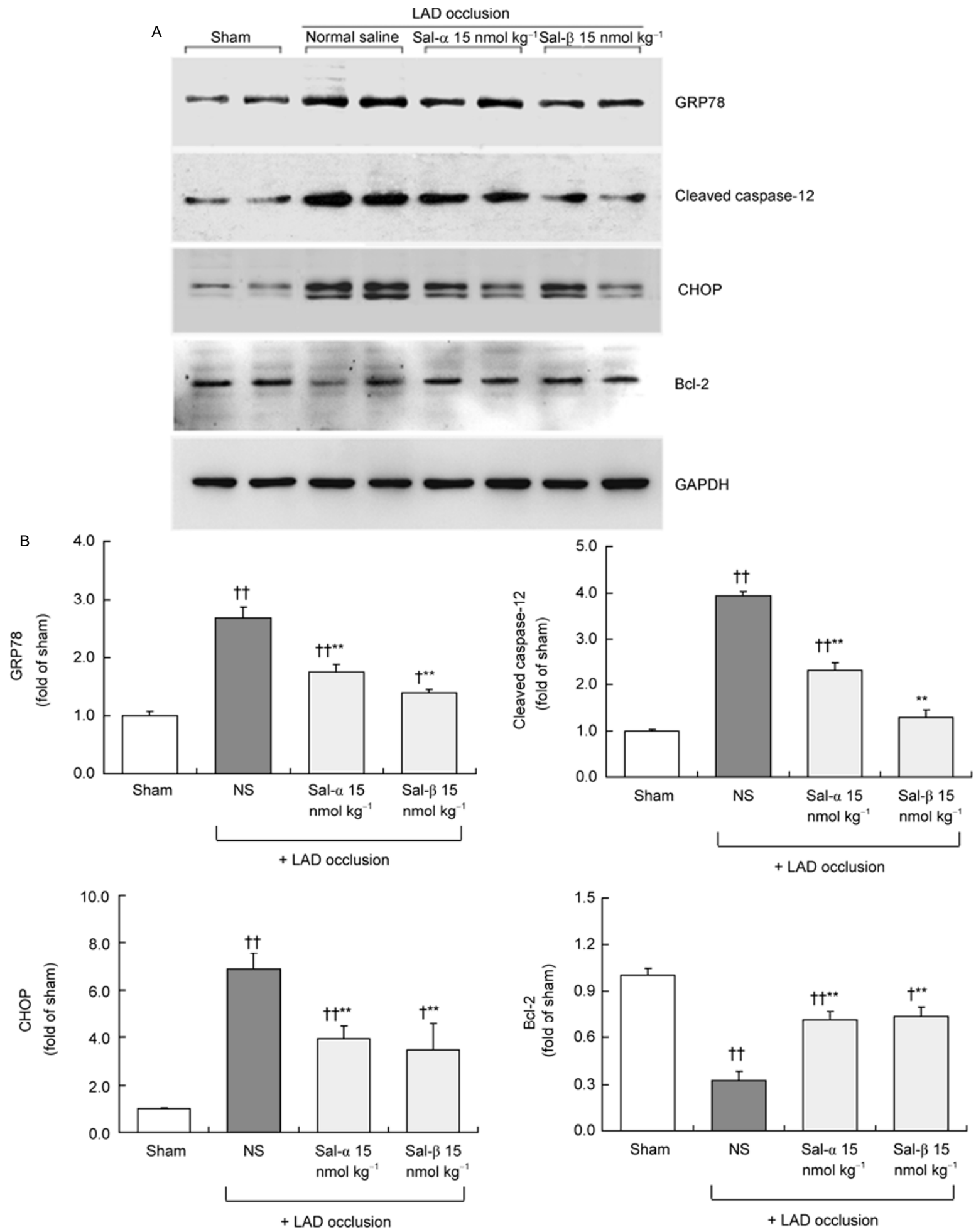

Figure 5 Expression levels of endoplasmic reticulum (ER) stress-associated proteins GRP78, active caspase-12, CHOP and Bcl-2 in sham control and ischemic myocardium after $2 \mathrm{~h}$ of LAD occlusion in rats. A, Representative images of Western blots for GRP78, cleaved fragments of activated caspase-12, $\mathrm{CHOP}$ and Bcl-2. B, Quantitative levels of the ER stress proteins. Values are means $\pm \mathrm{SD}$ ( $n=4$ for each group). $\dagger, P<0.05 ; \dagger \dagger, P<0.01 \mathrm{vs.} \mathrm{sham;} \mathrm{*,} P<0.05$; $* *, P<0.01$ vs. normal saline (NS)+LAD occlusion.

The novel finding of the present study is that both salusin- $\alpha$ and $-\beta$ can alleviate ER stress caused by myocardial ischemia, as evidenced by the inhibition of GRP78 and
CHOP overexpression, the suppression of caspase-12 activation, and the recovery of Bcl-2 expression (Figure 3). In addition, the myocardial apoptosis, infarct size and ventric- 

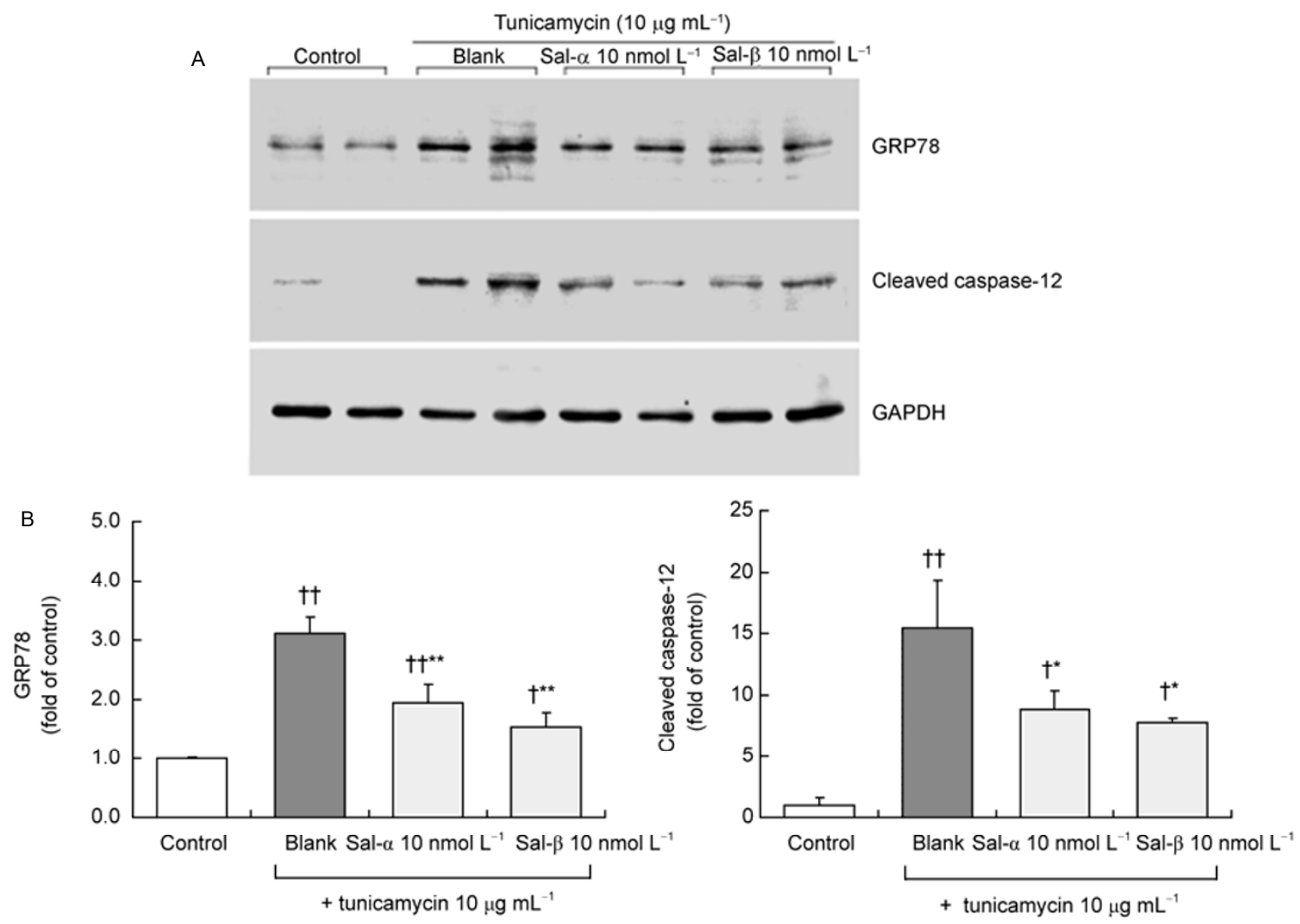

Figure 6 Salusins inhibited ER stress induced by tunicamycin in cultured rat H9c2 cardiomyocytes. A, Representative images of Western blots for ER stress markers, GRP78 and cleaved fragments of activated caspase-12. B, Quantitative levels of GRP78 and cleaved caspase-12. Values are means \pm SD ( $n=3$ for each group). $\dagger, P<0.05$; $\dagger, P<0.01$ vs. control; *, $P<0.05$; **, $P<0.01$ vs. tunicamycin.

ular arrhythmias caused by ischemia were reduced by salusins, and the heart function showed an improvement. The decrease in apoptosis was because of the suppression of ER stress-associated apoptosis, and the reduction of infarct size was presumably due to the inhibition of apoptosis, which is a significant feature of hypoxia and ischemia/reperfusion injury [21]. The attenuation of ischemic arrhythmias was probably associated with the improved homeostasis of calcium (a key mediator of arrhythmias), resulting from improved ER performance and a decrease calcium influx, as we described previously [8]. In accordance with reduced apoptosis, infarct size and arrhythmias, the heart function and hemodynamics in LAD-occluded rats was improved by salusins. These protective effects of salusins may represent a potential novel treatment for ischemic heart disease.

The present study did not observe changes in heart function or blood pressure in sham-operated rats receiving salusins. In accordance, two previous studies that used comparable doses of salusins did not see changes in heart function [7,23]. However, Shichiri et al. [1] observed bradycardia and hypotension in rats receiving relatively lower doses of salusins $\left(0.1,0.3,1 \mathrm{nmol} \mathrm{kg}^{-1}\right)$. These discrepancies may be related to the physicochemical features of salusins. The readily binding of salusins to commonly used polypropylene and glass tubes and syringes [3,24], which were also used by us, may inevitably cause loss of salusins. Despite the discrepancies of doses, the negative inotropic and chronotropic actions cannot be neglected when applying salusins.

In summary, the present study showed that salusin- $\alpha$ and $-\beta$ significantly reduced cardiac apoptosis, infarct size and ventricular arrhythmias, and improved heart function and hemodynamics in coronary-ligated rats. Concomitantly, salusin- $\alpha$ and $-\beta$ prevented the induction of an ER stress marker GRP78, reduced ER stress-associated proapoptotic proteins caspase-12 and CHOP, and recovered an ER stress-linked antiapoptotic protein Bcl-2, suggesting that salusins alleviated ER stress and ER stress-associated apoptosis in ischemic myocardium. Our results provide novel insights into the cardioprotective effects of salusins; however, the mechanisms underlying their inhibition on ER stress requires further study.

This work was supported by the National Basic Research Program of China (Grant Nos. $2006 C B 503807$ and 2009CB521902), the National Natural Science Foundation of China (Grant Nos. 30600763, 30870906, and 31071023), the Pujiang Project of Shanghai, China (Grant No. 08PJ14001), the Project Sponsored by the Scientific Research Foundation for the Returned Overseas Chinese Scholars, Ministry of Education of China (Grant No. [2008] 891), and the Fund for Outstanding Young 
Teachers in Higher Education Institutions of Shanghai, China (Grant No. [2009] 63).

1 Shichiri M, Ishimaru S, Ota T, et al. Salusins: newly identified bioactive peptides with hemodynamic and mitogenic activities. Nat Med, 2003, 9: 1166-1172

2 Sato K, Koyama T, Tateno T, et al. Presence of immunoreactive salusin- $\alpha$ in human serum and urine. Peptides, 2006, 27: 2561-2566

3 Sato K, Sato T, Susumu T, et al. Presence of immunoreactive salusin- $\beta$ in human plasma and urine. Regul Pept, 2009, 158: 63-67

4 Saito T, Dayanithi G, Saito J, et al. Chronic osmotic stimuli increase salusin-beta-like immunoreactivity in the rat hypothalamo-neurohypophyseal system: possible involvement of salusin- $\beta$ on $\left[\mathrm{Ca}^{2+}\right]_{i}$ increase and neurohypophyseal hormone release from the axon terminals. J Neuroendocrinol, 2008, 20: 207-219

5 Suzuki N, Shichiri M, Tateno T, et al. Distinct systemic distribution of salusin- $\alpha$ and salusin- $\beta$ in the rat. Peptides, 2011, 32: 805-810

6 Izumiyama $\mathrm{H}$, Tanaka $\mathrm{H}$, Egi $\mathrm{K}$, et al. Synthetic salusins as cardiac depressors in rat. Hypertension, 2005, 45: 419-425

7 Yu F, Zhao J, Yang J, et al. Salusins promote cardiomyocyte growth but does not affect cardiac function in rats. Regul Pept, 2004, 122: 191-197

8 Shi J S, Li D, Li N, et al. Inhibition of L-type calcium currents by salusin- $\beta$ in rat cardiac ventricular myocytes. Peptides, 2010, 31: 1146-1149

9 Talukder M A, Zweier J L, Periasamy M. Targeting calcium transport in ischaemic heart disease. Cardiovasc Res, 2009, 84: 345-352

10 Yan X H, Lin L, Pan Y X, et al. Salusins protect neonatal rat cardiomyocytes from serum deprivation-induced cell death through upregulation of GRP78. J Cardiovasc Pharmacol, 2006, 48: 41-46

11 Schroder M, Kaufman R J. The mammalian unfolded protein response. Annu Rev Biochem, 2005, 74: 739-789

12 Doroudgar S, Thuerauf D J, Marcinko M C, et al. Ischemia activates the ATF6 branch of the endoplasmic reticulum stress response. J Biol
Chem, 2009, 284: 29735-29745

13 Szegezdi E, Duffy A, O'Mahoney M E, et al. ER stress contributes to ischemia-induced cardiomyocyte apoptosis. Biochem Biophys Res Commun, 2006, 349: 1406-1411

14 Thuerauf D J, Marcinko M, Gude N, et al. Activation of the unfolded protein response in infarcted mouse heart and hypoxic cultured cardiac myocytes. Cir Res, 2006, 99: 275-282

15 Lin L, Yuan W J. Effects of different preproendothelin-1 mRNA anti-sense oligodeoxynucleotides on ischemic arrhythmias in rats. J Cardiovasc Pharmacol, 2002, 39: 590-599

16 Clark C, Foreman M I, Kane K A, et al. Coronary artery ligation in anesthetized rats as a method for the production of experimental dysrhythmias and for the determination of infarct size. J Pharmacol Method, 1980, 3: 357-368

17 Walker M J A, Curtis M J, Hearse D J, et al. The Lambeth Conventions: guidelines for the study of arrhythmias in ischemia, infarction and reperfusion. Cardiovasc Res, 1988, 22: 447-455

18 Johnston K M, Macleod B A, Walker M J A. Responses to ligation of a coronary artery in conscious rats and the actions of antiarrhythmics. Can J Physiol Pharmacol, 1983, 61: 1340-1353

19 Han C, Lin L, Zhang W, et al. Hyperbaric oxygen preconditioning alleviates myocardial ischemic injury in rats. Exp Biol Med (Maywood), 2008, 233: 1448-1453

20 Boyce M, Yuan J. Cellular response to endoplasmic reticulum stress: a matter of life or death. Cell Death Differ, 2006, 13: 363-373

21 Xu C, Bailly-Maitre B, Reed J C. Endoplasmic reticulum stress: cell life and death decisions. J Clin Invest, 2005, 115: 2656-2664

22 Groenendyk J, Sreenivasaiah P K, Kim do H, et al. Biology of endoplasmic reticulum stress in the heart. Circ Res, 2010, 107: 1185-1197

23 Gardiner S M, Keyte J, Bennett T. Reply to 'Salusins: newly identified bioactive peptides with hemodynamic and mitogenic activities'. Nat Med, 2007, 13: 661

24 Shichiri M. Reply to 'Salusins; newly identified bioactive peptides with hemodynamic and mitogenic activities'. Nat Med, 2007, 13: $661-662$

Open Access This article is distributed under the terms of the Creative Commons Attribution License which permits any use, distribution, and reproduction in any medium, provided the original author(s) and source are credited. 